\title{
AUTOMUTILATIONS PSYCHOTIQUES : ENTRE COUPURE ET ÉCRITURE
}

\author{
Gwénola Druel
}

ERES | « Cliniques »

2015/1 $\mathrm{N}^{\circ} 9$ | pages 40 à 61

ISSN 2115-8177

ISBN 9782749246857

Article disponible en ligne à l'adresse :

https://www.cairn.info/revue-cliniques-2015-1-page-40.htm

Distribution électronique Cairn.info pour ERES.

(C) ERES. Tous droits réservés pour tous pays.

La reproduction ou représentation de cet article, notamment par photocopie, n'est autorisée que dans les limites des conditions générales d'utilisation du site ou, le cas échéant, des conditions générales de la licence souscrite par votre établissement. Toute autre reproduction ou représentation, en tout ou partie, sous quelque forme et de quelque manière que ce soit, est interdite sauf accord préalable et écrit de l'éditeur, en dehors des cas prévus par la législation en vigueur en France. Il est précisé que son stockage dans une base de données est également interdit. 


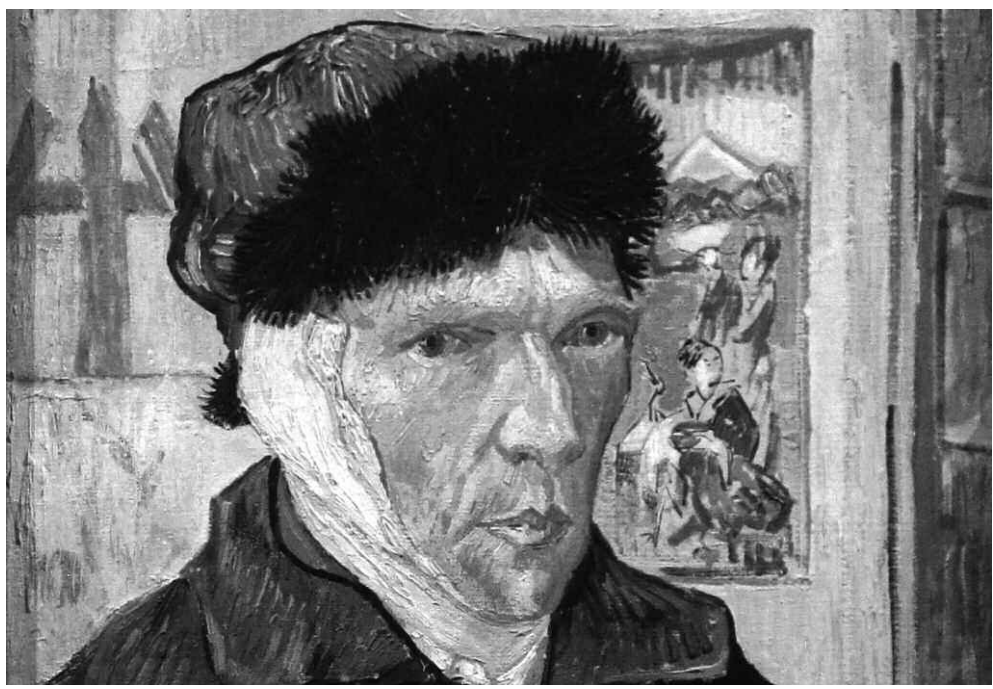

(c) Vincent Van Gogh, L'homme à l'oreille bandée.

«Bien souvent, ce geste répond au besoin de matérialiser une souffrance émotionnelle dans une douleur physique. » Gwénola Druel 


\section{Automutilations}

psychotiques :

entre coupure et écriture

Psychotic self-harm : between cutting and

writing

Gwénola Druel

ans un livre au titre évocateur Automutilation, M. Deville-

Cavellin (2005) raconte comment un jour, au lycée, alors que son mal-être prenait la forme de crises d'angoisse, elle s'est, dit-elle, « déclaré la guerre », et comment, cet acte « des plus malsains », «immoral», selon ses termes, devint vital pour elle. « Extériorisé le venin coulant en mes veines afin de me purifier de ces vices [...]. La saignée qui avait débuté par de petites écorchures durant l'enfance, des excoriations sans gravité, des ongles rongés jusqu'au sang, voire arrachés sans que je souffre véritablement de leur absence [...]. Ainsi, assise entre le radiateur et le mur, je levais ma manche gauche, armée d'un cutter spécialement emprunté pour l'occasion, puis, d'un geste rapide et saccadé, frappais contre ma chair décolorée pour me punir de cet état indélébile de morte vivante que je ne pouvais plus accepter. Rapidement, mon avant-bras se découvrait couverts d'entailles dont la cuisante douleur n'avait d'autre bénédiction que de me faire un bien indescriptible » (ibid.).

Gwénola Druel, psychologue clinicienne, psychanalyste en ESAT et en MAS, maître de conférences en psychologie clinique et psychopathologie, université Rennes 2, UFR Sciences humainesDépartement de psychologie EA 4050 . 
Toutes ces conduites automutilatoires placent le corps et l'affect au devant de la scène et ne peuvent laisser indifférents les champs de la médecine et de la psychopathologie. En effet, quel que soit le mode de surgissement, les automutilations interrogent les professionnels qui se retrouvent bien souvent désemparés, démunis, impuissants face à de tels passages à l'acte $\mathrm{d}^{\prime}$ « attaques du corps » dans le réel, auxquels ils ne peuvent donner sens et qui, parfois, ne font que s'intensifier si l'on intervient. Les registres de l'automutilation et de l'autodestruction sont alors bien souvent confondus. Comme le souligne C. Chiland (1984, p. 169-170), « ce qui nous inquiète, c'est la répétition, le caractère compulsif du geste, l'importance de la blessure allant jusqu'à un danger vital ». Quelle que soit la diversité des cas évoqués, l'automutilation vient signer un rapport singulier au corps propre; un mode bien spécifique $\mathrm{d}$ 'habiter la dimension humaine, en tant que ce phénomène automutilatoire témoigne d'un nouage particulier entre corps et langage. Le terme d'automutilation vient du grec autos, qui signifie soi-même, et du latin mutilare, sectionner, couper, retrancher. Dès 1909, dans sa thèse intitulée « De l'automutilation : mutilations et suicides étranges », à partir de nombreuses observations, pour la plupart des cas où le patient s'automutile en opérant une castration réelle de ses organes génitaux, M. Lorthiois (1909, p. 11) propose de définir l'automutilation comme une «atteinte portée à l'intégrité du corps ». Elle comprend, écrit-il, « toutes les pratiques entraînant des lésions des tissus et des organes », consistant « soit dans la blessure ou l'ablation totale ou partielle d'un organe ou d'un membre, du revêtement cutané [...] ; soit dans des manœuvres [...] pouvant compromettre [la] vitalité [du corps] et son bon fonctionnement sans que cependant elle ait été accomplie dans le but de se donner la mort » (ibid.). M. Strong évoque le cas d'un homme de 43 ans qui, bien que les automutilations qu'il s'inflige soient fort impressionnantes, ayant pu atteindre parfois des artères et nécessitant des transfusions sanguines aux urgences, il précise qu'il ne s'agit pas d'un acte suicidaire : «Je coupe secondairement pour la douleur, mais essentiellement pour le sang. » 
Regarder le sang jaillir le soulage, le «lave », dit-il. « Ça me purifie. [...] C'est comme si quelque chose de mauvais et de sale partait avec le sang. Alors, plus il y a du sang qui se répand, mieux c'est» (Strong, 2005, p. 11). Bien souvent, ce geste répond au besoin de "matérialiser » une souffrance émotionnelle dans une douleur physique (Corcos et Richard, 2006, p. 466) ; il apporte dès lors « une diminution de la tension interne, par l'extériorisation, l'authentification d'une douleur intérieure, non palpable » (Scaranozino, 2004, p. 26).

L'automutilation apparaît, notamment dans le champ des psychoses, comme une tentative du sujet pour retrancher, extraire sur son corps propre ce qui ne peut l'être par le langage. La coupure ne s'est pas déposée en lettres, mais ne cesse de se répéter comme marques, traces réelles sur le corps propre; un corps « en attente de retrouver dans l'Autre une demeure où se sentir hébergé, accueilli et reconnu » (Douville, 2009, p. 45). Considérer les automutilations en rapport à la dimension d'altérité, sans les réduire à des accès de fureurs autodestructifs, s'avère opérant. En effet, nous ne pouvons considérer le corps humain sans que se tresse un lien à une extériorité, sans que se façonne un rapport à l'autre. Ces phénomènes cliniques qui sont accueillis en institution ont leur racine dans une dimension relationnelle fondamentale. La relation avec le patient constitue l'enjeu même du traitement. Dès lors, précisément parce que la racine de l'insupportable, pour un sujet et/ ou pour son entourage, est de l'ordre du relationnel, la relation que le soignant engage avec le patient ne peut être quelconque. Certains auteurs confèrent à l'automutilation un statut au sein de la clinique de l'acte, dans la logique de l'acting-out. Dans ce cas, l'automutilation est adressée, elle est alors une tentative de mettre en lumière la souffrance, d'infléchir ou d'entamer l'Autre, de l'alerter, de susciter son intérêt. La valeur de monstration en jeu dans l'acting-out est de l'ordre d'un désir inconscient qui se manifeste, un act-out. Nous pouvons évoquer à ce propos certaines scarifications à l'adolescence notamment, qui ne sont pas exclusivement référées au champ des psychoses. Bien que dans la majorité des cas 
les scarifications soient pratiquées dans la solitude, B. Richard note, à juste titre, qu'elles sont bien souvent données à deviner à l'entourage par une manche discrètement relevée à moitié sur la plaie. Les scarifications ont pour visée de convoquer le regard de l'autre. Si ce qui est d'abord visé, c'est le soulagement des tensions internes, les bénéfices secondaires éventuels impliquent l'entourage, les autres patients et pairs - le plus souvent mis dans la confidence -, ou l'équipe soignante dont l'intérêt et la mobilisation sont obtenus par cet acte sans avoir à les demander. Le corps blessé est à la fois donné à voir et caché - pansement à demi-visible -, d'une manière qui peut être contredite par la parole; il montre à la fois la douleur et sa maîtrise, en laisse entendre le caractère involontaire en même temps qu'il expose la fondamentale contradiction des attentes envers l'objet; contradiction qui pourrait être formulée ainsi : "Si vous ne remarquez rien, c'est bien la preuve que vous n'en avez rien à fiche de moi [...]; si vous réagissez, sachez que je ne vous ai rien demandé et que vos interventions sont une intrusion inadmissible dans un domaine qui ne regarde que moi ; mon corps m'appartient » (Richard, 2005, p. 137). Les scarifications, de par les cicatrices que le sujet n'a de cesse d'entretenir, se présentent déjà comme des tentatives d'écriture à même la peau, tel un écrit indélébile sans qu'un sens soit pour autant recherché par le sujet. En effet, autant le patient peut évoquer son état de tension psychique et son besoin de s'en débarrasser, autant nous nous heurtons à de vives résistances dès que nous tentons de rechercher le sens d'un tel passage à l'acte. Ainsi, se poser la question de savoir si telle automutilation ou telle scarification effectuée sous les yeux de l'équipe peut être considérée comme un acting-out ou un passage à l'acte, dans l'optique de permettre au patient de penser de lui qu'il n'est pas si «fou » que ça, puisqu'il s'agit d'un acting-out, non seulement ne change rien à la dimension réelle de l'acte, mais « risque de détourner notre intervention de son point d'application utile » (Zenoni, 2009, p. 305). Insister sur cette distinction risque, en effet, d'orienter l'intervention sur l'acte lui-même : mettre des mots 
dessus, en parler, l'interpréter, avec les effets contre-productifs que l'on sait. Tandis que « prendre la mesure » du réel en jeu dans le passage à l'acte nous oblige à nous interroger et à intervenir sur le contexte de sa survenue, avec des conséquences apparemment plus favorables par rapport à son surgissement. D'autres manifestations automutilatoires surviennent en courtcircuit, rejetant tout appel à l'Autre, celles pour lesquelles le « laisser-tomber est le corrélat essentiel » (Lacan, 2004, p. 136); $c^{\prime}$ est le cas plus précisément de certaines automutilations psychotiques. Les automutilations qui s'inscrivent dans ce contexte relationnel peuvent revêtir un autre sens dès lors que quelqu'un s'en affecte ou y répond. R. Misès note, à juste titre, qu'à côté des automutilations graves présentées par certains autistes ou certains enfants psychotiques, des passages à l'acte qu'il qualifie d' " autoagressifs » peuvent survenir du seul fait qu'un adulte tente d'établir un contact avec l'enfant ou tente de modifier les protocoles de relation habituels entre l'enfant et lui (Misès, 1963, p. 1-78). Dans ce cas, l'automutilation surgit lorsque le sujet est « débordé », " envahi » par la présence et la demande de l'autre. C'est en cela que J. Aubry a opéré un pas dans l'abord du phénomène automutilatoire en proposant d'en considérer sa fonction de défense. Ces enfants réagiraient par ce repli du corps, et par cette façon de revenir à la matière même du corps, à une intrusion catastrophique de la présence d'autrui (Aubry, 2003, p. 135-173). Cela fut souvent observé, en particulier dans la clinique avec les enfants autistes. Inscrite dans une thématique délirante, l'automutilation apparaît comme un «mécanisme de défense contre l'angoisse massive d'anéantissement ou de morcellement » (Laxenaire et coll., 1984, p. 1284). Cette idée de « défense » à propos de l'automutilation suggère la mise en rapport du phénomène automutilatoire avec la fonction de coupure, la fonction de bord. Ces « conduites auto-offensives »- pour reprendre le terme de $\mathrm{H}$. Scharbach (1986) - font parfois suite à des conduites similaires dans l'enfance. Typiques ou atypiques, elles réalisent des actes de rupture. $S^{\prime}$ intéresser à ces moments de passages à l'acte automutilatoires donne des 
indications sur la façon dont le sujet tente de maintenir une séparation d'avec l'Autre ; séparation qui, dans la psychose, est insymbolisable. Dans le cadre d'un séjour en institution, il est dès lors opportun d'être attentif à tous les moments où la « séparation » peut être sollicitée. Il n'est pas rare, par exemple, que le risque de passage à l'acte augmente lors du retour définitif ou ponctuel dans le milieu de vie familial - même si le sujet ne peut envisager de vivre ou de passer un week-end ailleurs que là. De là aussi l'opportunité de la mise en place d'un lieu d'adresse régulièrement accessible, en dehors du cadre institutionnel où le sujet réside actuellement, afin qu'il puisse y avoir recours, indépendamment et au-delà de la période limitée du séjour.

Si l'on prend en compte les diverses situations cliniques où surgissent les automutilations, l'étude de ce phénomène est rendue délicate du fait que rares sont les associations verbales du sujet qui permettent de cerner ce moment automutilatoire. Elles substituent l'acte à la parole. Elles sont impulsives, transgressent les limites, visant un apaisement immédiat ou une recherche de contrôle. Les automutilations, tout comme les scarifications, se présentent comme exutoire corporel à la douleur psychique, ou encore visant une douleur physique pour faire oublier une angoisse insupportable, ou encore faire exister un corps vécu comme morcelé dans des cas de schizophrénie notamment. À l'extrême, le sujet psychotique vient témoigner de la chosification de son corps qu'il s'agit de maîtriser, voire de la réduction de son corps à l'état de cadavre, voire encore d'une modification pour « se faire » un corps qui tienne. Dans la clinique des psychoses, les automutilations témoignent bien souvent d'une profonde altération dans la perception du corps. Ces passages à l'acte surgissent là où les mots manquent, là où le réel du corps ne peut se dire avec des mots. Le réel est ici défini comme l'impossible à dire ou à signifier, la catégorie du réel renvoie à ce qui ne peut être complètement symbolisé dans le champ de la parole. Le recours aux automutilations, aux scarifications, dans ces versions psychopathologiques, renvoie très précisément à une clinique 
du réel. Il s'avère dès lors nécessaire d'interroger les coordonnées du déclenchement de tels passages à l'acte et de procéder à un examen minutieux des modalités de leur apparition, voire de leur surgissement. Ce repérage clinique nous donne des indications précieuses sur l'insupportable auquel le sujet a affaire et ce à quoi répondent les automutilations ; cela est au commencement d'une prise en charge possible.

L'adolescence est particulièrement propice à cette rencontre du sujet avec le réel, le réel de l'adolescence, celui qui est incontestablement introduit par le pubertaire (transformations physiques, physiologiques et hormonales de la puberté) dans une étrangeté (Unheimliche) d'autant plus inquiétante qu'elle habite le sujet. La perte de repères, de limite, qui découle de la puberté, le sentiment d'étrangeté sont autant de facteurs qui favorisent des pratiques singulières impliquant le corps. Ce corps qui change induit chez l'adolescent, l'adolescente, sidération, angoisse, voire dépersonnalisation. Ce temps de réactivation pulsionnelle vécu comme imposé (parfois de manière persécutrice) avec son lot d'inquiétude, d'angoisse et de défenses multiformes, oblige chaque jeune à reconsidérer le regard qu'il (elle) porte sur lui (elle)-même, mais aussi celui qui est désormais porté à ce corps désirant qu'il ne reconnaît plus. Tel est ce décalage entre l'image du corps - du temps de l'infantile - et l'image spéculaire que l'on retrouve dans certains évitements du regard comme dans ce souci du moindre indice de défaut corporel. Cette « angoisse » signale la rencontre avec ce qu'a de réel le corps aux prises avec cette part du vivant, au-delà des signes de l'organisme. Cette violence à l'endroit du corps, parce qu'elle est «en excès » et déborde le sujet, se présente en deçà de toute possibilité énonciative. Il est alors des cas où certaines automutilations à l'adolescence peuvent présider à des entrées dans la psychose (Delaroche et Mouras, 2004). Le cas d'Angélique (Bouillot, 2003, p. 135-140) est, à ce propos, tout à fait singulier quant à une clinique de l'automutilation psychotique et sa prise en charge institutionnelle. 
AUTOMUTILATIONS ET « DÉROBOTISATION »

Angélique est une jeune fille âgée de 20 ans, accueillie depuis cinq ans dans l'institution. Depuis son plus jeune âge, Angélique fait d'incessants allers et retours entre sa famille et de nombreuses institutions, hôpitaux et familles d'accueil. Son placement actuel en institution est censé la séparer de son milieu familial dans lequel elle ne peut séjourner plus de quelques mois sans être au plus mal. Toutefois, ce placement n'a pas d'emblée l'effet d'apaisement escompté. Elle ne peut s'empêcher de s'automutiler dès que lui vient un vif sentiment d'indignité qu'elle éprouve à se sentir réduite au statut de « robot de sa mère », idée qui lui est venue vers sa treizième année. 13 ans est bien l'âge auquel remonte, selon la mère d'Angélique, « un moment de bascule », moment de déclenchement des troubles psychotiques actuels. Alors que sa fille jusqu'alors ne formulait aucune demande, elle a commencé à lui adresser d'incessants reproches, ceux de l'avoir réduite à l'esclavage, de l'avoir robotisée, de l'avoir affamée, de lui refuser toute place dans la famille. C'est à cette époque qu'apparaissent des douleurs et les troubles de l'image du corps. Un jour, alors que le père d'Angélique cherche à la défendre, la mère dira : " mais on ne frappe que sur un robot »; parole, prise au pied de la lettre, qui raisonnera comme une certitude pour Angélique, celle de n'être qu'un robot pour sa mère. Angélique attribue son admission en institution à une « complication avec sa mère ». Elle nomme sa souffrance, son « mal de mère », une blessure profonde en permanence béante qui prend une dimension très réelle dans l'automutilation. Ce « mal de mère » n'a rien d'une métaphore mais vient dire les douleurs très vives qui l'accablent. D'être séparées ou réunies est vécu par l'une et l'autre très douloureusement. Sa mère la considère comme «morte » quand elle n'est pas à ses côtés. Elle sait qu'elle est ce qui manque à sa fille et ne peut admettre l'idée que le souhait de se tenir écartée d'elle puisse venir spontanément à sa fille. Son point de certitude concerne la manipulation dont sa fille est victime et qui consiste à l'éloigner 
d'elle. La prise en charge institutionnelle s'avère donc d'emblée délicate. Dans le cadre institutionnel, les remontrances les plus vives adressées à Angélique restent lettre morte, tandis que des paroles apparemment anodines déclenchent mal-être profond et délabrements corporels. Les tentatives de séparation avec le milieu familial échouent. Angélique n'a de cesse de téléphoner à sa famille pour se plaindre de l'institution jusqu'à ce que ses parents viennent la retirer de force. Et, aussitôt arrivée chez ses parents, elle ne cesse d'exiger une réintégration immédiate dans l'institution.

Dans ces allers-retours se succèdent, entre la mère et la fille, " déclarations d'amour » et « dénonciations délirantes », tout cela ayant pour effet de produire chez Angélique des douleurs dans le corps et un sentiment de dévitalisation. Le souhait exprimé par Angélique est toutefois celui d'être éloignée de sa mère, mais l'être physiquement ne suffit pas à l'apaiser ; car ce qui détermine l'alternance des phénomènes douloureux dans le corps est non pas tant la présence ou l'absence physique de la mère que la prégnance ou non des «pensées de mère », comme les nomme Angélique. Ces pensées s'imposent à elle et se présentent comme des paroles quasi-hallucinatoires. De rares moments de répit se font jour lorsqu'une séparation s'opère d'avec les «pensées de mère ». C'est pour traiter les douleurs insupportables et les hallucinations qu'Angélique s'entaille les bras ; entailles qui s'arrêtent " au premier sang ", dit-elle. L'apaise-ment vient lorsque le sang s'écoule et que la douleur de l'entaille chasse celle provoquée soit par les paroles de la mère, soit par les "pensées de mère ». Si quand, en présence de sa mère, Angélique parvient à entendre le son de sa voix mais pas les paroles qu'elle profère à son encontre, elle se sent alors plus apaisée. Et quand elle n'y arrive pas ou plus, ce sont les entailles qui servent de défense à ses douleurs.

La prévalence du lien à la mère était telle que s'est posé dans l'institution la question de savoir ce qui pourrait venir s'introduire entre elles deux. L'entaille semble introduire une coupure réelle sur son corps comme tentative de faire barrage à l'envahissement des «pensées de mère ». Ici, les entailles 
laissent entendre que le terme d'automutilation doit être pris, non pas dans le sens d'une atteinte du corps visant à le mutiler, à le trouer, à l'amputer de quelque chose qui serait de trop, mais bien au contraire dans le sens de rajouter par cette opération quelque chose qui n'a pu se symboliser, c'est-à-dire une zone où pourrait se localiser cet envahissement du corps. L'acte de coupure est une tentative d'introduire un « reste » qui ne peut être symbolisé, hors signifiant, hors corps. L'image du corps est supportée par ce reste qui décomplète, introduisant un manque, un " pas-tout », réfrénant la part de jouissance du vivant. L'entaille la « dérobotiserait », non pas parce qu'à voir son sang couler elle vérifierait qu'elle n'est pas un robot, mais parce qu'elle l'empêche d'éprouver son corps comme complètement envahi. L'institution s'appuiera sur cette indication dans le traitement de l'automutilation afin de permettre à Angélique de trouver une autre solution que les entailles pour se séparer des paroles et des "pensées de mère ». L'équipe soignante ne s'est pas attelée à lui interdire à tout prix la pratique de ses entailles, car cet interdit avait l'effet de relancer ses passages à l'acte; mais il lui sera proposé des activités centrées sur les «soins de beauté ». Prendre ainsi soin d'elle, de son corps, porter une attention particulière aux vêtements ont permis une certaine restauration imaginaire du corps incitant Angélique à beaucoup moins l'attaquer dans le réel.

Son rapport au corps est néanmoins fragilisé depuis qu'elle a un « amoureux ». Elle entretient avec ce garçon un lien calqué sur le modèle du lien à sa mère. Elle est tout pour lui et il ne supporte pas qu'elle lui échappe, au point qu'il s'est scarifié son prénom sur le torse. Il la menace de se tuer si elle le quitte. Angélique elle-même repère qu'il parle comme sa mère. Quand elle pense à lui, elle pense aussi à ses parents. Elle lui demande de ne pas s'approcher d'elle et se sent salie par ses avances. L'équipe aide Angélique à trouver des moyens de maintenir ce garçon à distance. Toutefois, elle peut dire qu'elle est « une fille qui a un amoureux». Angélique a également trouvé à s'assurer d'une présence constante de la voix sous la forme du brouhaha ambiant et anonyme de l'institution qui la 
détend au point de trouver le sommeil au beau milieu des pièces communautaires, ce à quoi l'équipe consent. Le suivi thérapeutique consistera, notamment, à permettre une « séparation » d'Angélique avec les pensées qui l'envahissent en soutenant un travail sur la langue et la lettre. Depuis quelque temps, Angélique marque en effet un intérêt pour le langage. Elle commence notamment à répertorier, à inventorier les tics de langage, les thèmes de prédilection et le style propre à chacun des soignants, en tentant à chaque fois qu'elle s'adresse à eux de leur parler dans « leur langue ». Elle a fait à l'occasion des efforts importants pour parler à chacun d'eux. Sa tentative rencontre toutefois un point de limite quand elle se retourne en soumission aux paroles qu'elle reçoit comme "persécutives » de ceux à qui elle essaie de parler. Ce travail sur la langue a néanmoins l'intérêt de la détacher provisoirement de la fixation à la formule « je suis le robot de ma mère » ou aux « pensées de mère » qui avaient pour effet de déclencher ses passages à l'acte automutilatoires. Là où la rupture est au premier plan dans la psychose - ce dont témoignent notamment les passages à l'acte -, ce que fait valoir ici le travail institutionnel est une pratique du lien, mais un lien « réglé » sur ce qui est supportable pour le sujet.

Le cas d'Angélique n'est pas sans évoquer, même s'il s'en distingue, le cutting, forme épurée de scarification. En effet, le cutting désigne l'envie irrésistible de se couper, d'inscrire à même la peau des traces réelles par des cicatrices indélébiles. C'est une " pratique impérieuse et répétitive, ne recouvrant pas pour autant des problématiques homogènes, et ne possédant, avant toute analyse du cas, aucune valeur pathognomonique » (Trichet et Lévy, 2008, p. 399). Une telle définition n'est pas sans revêtir une pluralité des tableaux cliniques. Nous pouvons toutefois y entrevoir chez certains sujets la fonction singulière dans l'économie de la jouissance. Carla est présentée par M. Strong (2005, p. 175) comme une « cutter extraordinairement compulsive », pouvant se couper deux à trois fois par jour, où qu'elle soit. Elle entretient fréquemment ses plaies en luttant contre la cicatrisation et en 
essayant de les infecter. Se couper fait réponse pour elle quand elle est trop joyeuse, trop excitée, ou bien au contraire déprimée. Le cutting vient alors tempérer ses humeurs et la « ramener sur terre »- comme « une pilule magique » (ibid.), dit-elle - dans une singulière sensation d'apaisement d'un corps qu'elle perçoit comme douloureux et énigmatique. Pour certains sujets, le cutting produit un apaisement singulier dans leurs rapports avec le corps propre, et apparaît également comme une réponse à l'angoisse et à la perte. Le cutting, les scarifications, mais aussi d'autres pratiques d'inscription réelle sur la peau comme les tatouages, les piercings, les implants peuvent, chez certains sujets psychotiques, être considérés comme une modalité possible de se stabiliser activement hors déclenchement clinique.

Aussi malheureux soient-ils, la portée résolutoire, du moins libératrice, des passages à l'acte automutilatoires est indéniable. Les automutilations, les scarifications et tout autre marquage corporel, à des degrés variables, sont autant de tentatives « autothérapeutiques ». Il s'agit dès lors de s'orienter à partir de cette indication afin de permettre au sujet psychotique de trouver une autre solution pour faire avec l'insupportable qu'il rencontre ; une coupure qui soit autre qu'un retour réel sur le corps. $M^{\text {lle }} \mathrm{B}$. est une patiente de 30 ans fréquemment hospitalisée (Doucet, 2011, p. 55). Chez elle, en famille d'accueil, dans l'unité $\mathrm{d}^{\prime}$ hospitalisation, $\mathrm{M}^{\mathrm{lle}} \mathrm{B}$. se coupe très souvent et est transférée au CHU. Ses passages à l'acte sont une percée libératrice, ce n'est pas un appel ni un désir de mourir. Elle ne peut rien dire sur ce qui la menace. Ses passages à l'acte ne sont pas une forme de verbalisation. C'est plutôt une absence de verbalisation. Ses passages à l'acte la soulagent. Avant ceux-ci, Mlle B. interpelle l'équipe pour dire que son œil va mal, qu'elle a mal à la tête ou aux jambes. Elle dit : « Je ne sais pas quoi faire. » C'est une phrase interrompue qui se boucle par le passage à l'acte automutilatoire. Au-delà d'avoir un corps qui part par petits bouts, lors de ses passages à l'acte, elle a affaire avec un réel hors langage. La pousser à verbaliser la précipite vers un vide de sens. Il faut panser ses plaies plutôt que d'aller vers le sens. 
$M^{\text {lle }}$ B. peut dire que lorsqu'elle est seule, elle pense à ses passages à l'acte : «Je me creuse la tête, puis je me creuse mon corps. » À la suite de ses passages à l'acte, souvent elle fait des reproches, insulte ; elle place la faute du côté de l'équipe, ce qui l'éloigne quelque temps de ses passages à l'acte. Répondre en urgence lorsqu'elle présente une partie de son corps qui va mal, mais en faisant intervenir un autre interlocuteur peut aussi introduire un léger écart. L'équipe soutient également $\mathrm{M}^{\mathrm{lle}} \mathrm{B}$. lorsqu'elle s'achète des bagues pour chaque doigt ou de multiples piercings qui viennent tenir son corps. Son traitement par la « coupure » est soutenu quand l'équipe élabore pas à pas un projet avec elle pour qu'elle soit un temps à l'hôpital, un temps en famille d'accueil, un temps chez sa mère... À la place de l'entaille qu'elle se fait sur le corps est introduite une " coupure » des lieux où chacun d'entre eux n'est pas tout pour elle. Ce qui fait alors continuité pour $\mathrm{M}^{\mathrm{lle}} \mathrm{B}$., c'est son ordinateur portable qui circule d'un lieu à l'autre et les lettres qu'elle adresse à l'équipe.

Avant même de viser à traiter le sujet, l'institution existe pour l'accueillir, le mettre à distance, l'aider, l'assister ; l'institution est une « nécessité sociale » (Zenoni, 2009, p. 18). C'est la nécessité d'une réponse à des phénomènes cliniques, tels certains états de la psychose, certains passages à l'acte, certains états de délabrement physique qui peuvent amener le sujet vers l'exclusion sociale ou vers la mort. L'institution vient à la place du lien social devenu impraticable; préalable nécessaire à tout traitement possible de la psychose.

\section{L'ÉCRITURE : UNE SOLUTION « AUTOTHÉRAPEUTIQUE » ?}

J'évoquerai ici le cas de Fabien, 30 ans, qui est exemplaire quant à la fonction particulière de l'automutilation, et singulier quant aux effets d'apaisement que produira, chez ce sujet, sa solution par l'écriture.

Je rencontre Fabien à sa sortie d'hospitalisation, deux semaines après qu'il a tenté de se suicider en absorbant une forte dose de médicaments. Il a été diagnostiqué, lors de son hospitalisation, 
une schizophrénie avec épisode dépressif. Le mal-être de Fabien dure depuis l'enfance, mais tout s'est accéléré ces derniers mois après le suicide d'un ami proche de la famille ; celui aussi, par pendaison, d'une de ses amies, et la rupture, depuis plusieurs semaines, avec sa petite amie rencontrée sur Internet. Dans ces pertes et ruptures successives, l'ombre de la mort se présentifie réellement. Un souvenir lorsqu'il était âgé de 4-5 ans vient articuler le « laisser-tomber » de l'Autre à la mort : «J'ai failli me noyer dans une chute d'eau, j'avais peur et mes parents n'ont pas bougé. J'ai pensé que j'allais mourir. J'appelais à l'aide et mes parents s'en foutaient, je ne comptais pas. Ils m'entendaient mais ils n'ont pas bougé. » Un autre événement marquant de son enfance vient pour Fabien sceller, comme un pacte inexorable, la perte et la mort. Lorsqu'il était âgé de 6 ans, son grand-père meurt brutalement : " C'était un grand-père merveilleux et $\mathrm{du}$ jour au lendemain, il s'est retrouvé sous une pierre tombale. C'est là que tout a commencé. Je me suis rendu compte qu'on était mortel et qu'on allait perdre tous nos êtres chers. »

Les liens que Fabien tente de tisser avec des jeunes femmes de son âge, via les réseaux sociaux, le retiennent quelque temps ; mais l'amitié, l'amour et la mort semblent se nouer inexorablement. C'est sur ces mêmes réseaux sociaux que Fabien est en contact, depuis plusieurs mois, avec une jeune fille, S., avec qui il débutera, dit-il, « une relation très virtuelle ». Très vite des sentiments amoureux surviennent et l'envahissent. Il lui voue " un amour sans borne », précise-t-il. Il lui avoue néanmoins qu'il communique avec d'autres filles sur Internet. Là, où jusqu'alors elle le comprenait, ne le jugeait pas, cet aveu eut l'effet, chez cette jeune fille, de mettre fin à leur relation. Ce fut, dit-il, l'élément déclencheur qui le mena à sa tentative de suicide. Depuis cette rupture, Fabien ne peut plus « toucher » son ordinateur ; " j'essaie de trouver autre chose pour m'occuper, j'ai le goût à rien, je n'ai plus de passion. Je ne me sens pas exister. Je me retrouve face à un vide». Avant cet épisode, Fabien s'intéressait à la « création graphique » sur ordinateur : « Je voulais créer un monde le plus cohérent possible avec ses 
logiques, un univers dans lequel on puisse vivre dedans. » Par la création graphique, Fabien semble chercher à mettre de l'ordre, à inscrire une cohérence dans un monde immaîtrisable où chacun peut mourir à tout moment et le laisser en plan. Je prendrai notamment appui sur cet intérêt de Fabien pour la création graphique afin de soutenir sa recherche d'une solution pour vivre dans un monde supportable.

Depuis sa rupture avec cette jeune fille, il pense beaucoup à elle, à ce qu'ils se disaient via le forum, suscitant chez lui beaucoup d'angoisse. Des phénomènes de corps apparaissent alors : « Je suis bloqué au niveau de la poitrine. J'ai l'impression d'avoir du plomb fondu dans les veines; tout mon corps me fait mal, j'ai une espèce d'hypersensibilité. Ça commence dans le bras gauche et après ça m'envahit au fur et à mesure. » Il essaie de se contrôler, mais une forte douleur envahit son corps et des « crises de larmes » surviennent. C'est dans ces moments de profond désarroi que Fabien s'entaille le haut du corps (bras gauche, épaule et poitrine) avec un cutter : « C'est, dit-il, pour effacer la douleur psychologique par la douleur physique. Le principe, c'est que ça saigne, que ça fasse mal. Cette douleur physique me réconforte, quand j'ai mal là, je n'ai pas mal ailleurs. » Pendant cette période, Fabien arrêtera aussi de manger afin de susciter la « douleur de la faim » pour masquer la « douleur psychologique de la tête et du corps ».

De cette douleur dans la tête, Fabien peine à pouvoir en dire quelque chose : « Je n'arrive pas à la définir. Je n'ai pas de mots. Je ne sais pas ce que c'est, mais c'est une douleur qui me prend le corps et l'esprit, qui me brûle l'esprit et le corps. Au mieux, je m'écroule et je pleure. Au pire, je fais des crises délirantes où j'essaie de me calmer en me remettant à la peinture. J'essaie de sortir de cette douleur en la mettant en dehors de moi, sur la toile. Je rentre dans un état de transe, je me taillade le corps, je peins des symboles sur mon corps. J'ai un besoin viscéral d'expression, c'est vital. J'arrive pas à expulser toutes mes pensées, il faut que je trouve une autre méthode pour matérialiser mes idées. » Fabien cherche à extraire de son corps cette douleur insupportable qui trouve à se représenter 
dans sa tête par des images qui lui font peur. Il cherche un moyen, non pas de communication « de peur de devenir fou », mais «d'expression» de tous ses sentiments emmagasinés, douloureux dans son corps. Il veut les extraire de lui pour en faire quelque chose d'autre du côté d'une «création ». Cette indication précieuse qu'il me donne m'orientera : ne pas l'inciter à trop en dire avec des mots - ce qui a l'effet de susciter son angoisse -, mais lui permettre de trouver un moyen d'expression autre que les mots, autre que les automutilations, pour extraire cette douleur insupportable du corps. Je l'invite alors à ce que nous trouvions ensemble cette autre méthode d'expression qui ne mette pas en péril son corps et sa vie.

Dans les séances qui vont suivre, Fabien me confie qu'il commence à ressentir le besoin de créer, différemment de ce qu'il faisait avant; séances qui s'inaugureront par cet énoncé : «Mon sens créatif commence à me chatouiller un peu. Je suis à la recherche de cette manière de s'exprimer. Je veux développer un moyen d'expression. » Fabien consent à mon aide dans sa nouvelle recherche créative. Malgré des moments où il se sent angoissé, notamment lorsqu'il se retrouve au milieu d'une foule, il poursuit la recherche d'une solution qui lui permettrait d'extraire ses idées qui ont un effet douloureux direct sur le corps. Il y a aussi ce jour où il se taillade le bras gauche et la poitrine à l'emplacement du cœur, après que la jeune fille, qu'il dit toujours aimer, ne réponde pas à ses messages : «Elle a fait une croix sur moi, elle m’a rayé de sa vie et moi j'ai fait pareil sur mon corps. Ce qui me blesse le plus, c'est son silence. Je me coupe la peau, ça me fait mal physiquement pour oublier mes angoisses. Je pense à ça et ça m'empêche de penser à autre chose. » Ce qui se joue de cette rupture avec cette jeune fille, de ce laisser-tomber, passe directement sur le réel de son corps sans une possible symbolisation. La « blessure » du silence prend une valeur très réelle par la blessure de son corps. Fabien veut me montrer ses blessures ; je refuse, et lui dis de trouver comment écrire et « matérialiser » ses pensées autrement qu'en blessant son corps. Parler lui étant parfois douloureux, l'écriture lui permettrait de 
dire autrement, sans engager une prise de parole et tout en déposant une trace réelle ; une coupure d'avec ce qui lui est insupportable, par l'écriture des mots.

Fabien continue à venir me voir pour mettre de l'ordre dans ses idées de créativité qui se bousculent dans sa tête, à la recherche de cette nouvelle méthode pour exprimer autrement sa souffrance intérieure. Un jour, il ne viendra pas à notre rendez-vous, c'est sa mère qui m'appelle, inquiète car il ne s'est pas levé. Je demanderai à parler à Fabien, il y consent. Il me dit se sentir mal, que la nuit a été dure car la veille il a eu « la sensation très précise du dernier baiser » échangé avec la jeune fille rencontrée sur Internet. Contrairement aux autres fois, Fabien n'utilisera pas un cutter : "J'essaie de me raisonner, de casser cet effet d'enchaînement. J'ai réussi à trouver des choses pour exprimer mon mal. Pour éviter de faire avec un cutter, j'ai pris un feutre noir et je me suis fait des marques et des dessins sur le corps. J'ai marqué aussi mes pensées négatives sur mon corps. Toutes mes pensées, je les ai marquées sur moi. Il fallait que j'exprime, que ça passe de l'intérieur à l'extérieur de mon corps, ça m'a calmé. » Je soutiendrai cette "invention » de Fabien, celle d'écrire sa souffrance avec des dessins, des mots à même la peau. Avec ces dessins et ces écritures sur le corps, nous ne sommes pas loin, du fait de leur caractère indélébile, de la fonction d'empreinte de certains tatouages; tentative de symbolisation par l'inscription sur la chair, " parure de peau pour faire peur ou se protéger [...] cette cicatrice imprimée, parée, sépare en introduisant dans le contact visuel un écart entre soi et l'Autre, le tatouage procédant sur un mode de communication visuelle» (Estellon, 2004, p. 147).

Une " communication visuelle», c'est bien ce que recherche Fabien. Il se souvient d'un voyage qu'il a fait à New York avec ses parents, il a eu une "révélation » alors qu'il visitait un musée d'art moderne. «Quand je me suis retrouvé devant les œuvres réelles, j'ai senti beaucoup d'émotions, je ressentais le peintre dans l'œuvre, il y avait de l'expression et ça m'a plu ; moi qui n'arrive pas à m'exprimer. [...] Pour moi, le principe 
de l'art, ce sont les émotions. Tant qu'on a des émotions, on peut en parler. » Si écrire sur papier l'a aidé un temps, ce n'est pas cette solution qu'il retiendra, car il lui faut trouver un mode d'expression, un «support » pour exprimer les images qu'il a dans la tête et qui lui procurent des sentiments. C'est plus du côté du dessin et de la création graphique sur ordinateur qu'il cherche une solution, en tant que la création, pour lui, «c'est l'idée de se purger plutôt que de créer. J'ai des sensations, des idées dans la tête, il faut que je crée quelque chose pour les sortir. La création, c'est se vider la tête et non pas partir de rien pour créer quelque chose. Me vider la tête me soulage parce que ces idées ne sont plus actives, elles ne sont plus en phase de développement. Je dois trouver un moyen pour les exprimer plus facilement, pour que ce soit moins douloureux et ne pas attendre d'arriver à la phase critique. Je n'arrive pas à exprimer mes idées au fur et à mesure. Il faudrait que j'arrive à faire des créations secondaires, ce serait palliatif. » Par sa recherche de «créations secondaires », Fabien essaie d'introduire des temps de scansion, des « coupures », dans un défilé de pensées qui sinon ne peuvent s'arrêter.

Fabien, dans son rapport à la création, indique très justement, ce qui spécifie la logique créative du sujet psychotique : il ne crée pas autour d'un vide, ce n'est pas le manque qui suscite la production, mais un trop-plein de pensées, de jouissance. Sa création consiste fondamentalement, pour lui, en la recherche d'un travail d'ex-pression par lequel s'opérerait une contention de la jouissance. À mesure que se précise pour Fabien une solution pour s'exprimer, les automutilations disparaissent. Il y a, malgré tout, des moments où il se sent moins bien, notamment lorsqu'il est en contact sur Internet avec des jeunes filles « victimes d'incestes ou de viol ». Même s'il me dit ressentir beaucoup de douleur pour ces jeunes filles, cela ne l'envahit plus. « J'arrive à me limiter, mais c'est pour mieux les aider. » Il se révolte parfois lorsque la parole de ces jeunes filles n'est pas prise au sérieux. Il commence à reprendre contact avec quelques amis, avec qui il sort le week-end. Là où l'être cher 
pouvait disparaître à tout moment, où lui-même pouvait s'extraire de la scène du monde, Fabien commence à tisser des liens et à trouver place dans un monde plus apaisé sans que les automutilations surviennent comme unique réponse à son mal-être profond.

\section{CONCLUSION}

La clinique de la psychose nous invite à envisager les automutilations comme des «solutions » a minima, tant d'une coupure que de résorption de l'aspect infini que comporte l'angoisse du sujet psychotique, qui le mène à perdre, avec la dépersonnalisation, le sentiment même de l'existence. L'atteinte faite à l'organisme devient, par la perte effectuée réellement, l'ultime garant d'une vie encore possible. Ce serait donc une manière de produire un substitut salutaire de l'extraction de l'objet non opérée symboliquement. Si les modes d'autotraitement sont variables et évolutifs dans le temps, et selon les sujets, ils ont pour point commun de viser un traitement du réel insymbolisable auquel a affaire le sujet psychotique. Toutefois, la visée de notre pratique clinique doit être de permettre ou de favoriser un autre « traitement » que celui qui pousse le sujet psychotique à couper, découper, se couper, se taillader..., dans le registre du réel ; en somme à « favoriser le recours à une forme de réel plus symbolique » (Zenoni, 2009, p. 301), une forme de réel moins ravageante, moins nocive, plus compatible avec le lien social, voire plus créative, que le réel du passage à l'acte. L'écriture à même la peau serait dès lors de l'ordre d'une solution du côté d'un réel plus symbolique. Elle est une tentative pour canaliser l'insupportable douleur émotionnelle interne. Le corps du sujet psychotique, envahi par une jouissance débordante, trouverait un apaisement par le truchement de l'écriture. Toutefois, ce travail d'écriture est toujours, pour le sujet psychotique, à réitérer. L'issue des efforts créatifs reste en effet incertaine, mais ils s'ancrent dans l'intuition de l'existence d'une tempérance de la jouissance inhérente à la production d'œuvres. 


\section{BIBLIOGRAPHIE}

Aubry, J. 2003. Psychanalyse des enfants séparés, Paris, Denoël.

BouIllot, P. 2003. «Se couper du corps de l'Autre », Les feuillets du Courtil, $\mathrm{n}^{\circ} 21$.

CHILAND, C. 1984. «L'automutilation : de l'acte à la parole », Neuropsychiatrie de l'enfance, 32, 4 .

CORCOS, M. ; RICHARD, B. 2006. «L'émotion mutilée : approche psychanalytique des automutilations à l'adolescence », La psychiatrie de l'enfant, vol. 49, 2. Delaroche, P. ; Mouras, J.-P. 2004. Peut-on devenir fou ?, Toulouse, érès.

Deville-CaVellin, M. 2005. Automutilation, Paris, Nantes, Éditions Amalthée. DOUCET, C. (sous la direction de). 2011. Le psychologue en service de psychiatrie, Issy-les-Moulineaux, Masson.

Douville, O. 2009. «Considérations psychanalytiques à propos de l'automutilation », Santé mentale, $\mathrm{n}^{\circ} 143$.

ESTELLON, V. 2004. «Tatouage sur corps ou l'envers de l'expression », Champ psychosomatique, $\mathrm{n}^{\circ} 36$.

LACAN, J. 2004. Le Séminaire, Livre X (1962-1963), L'angoisse, Paris, Le Seuil. LAXENAire, M. ; Millet, F. ; WestPhal, C. 1984. « Les automutilations : frontières et signification », Annales médico-psychologiques, vol. 142, n 10.

LORTHIOIS, M. 1909. De l'automutilation: mutilations et suicides étranges, thèse d'exercice : Médecine, Lille.

Mises, R. 1981. Cinq études de psychopathologie de l'enfant, Toulouse, Privat.

MISÈS, R. ; BARANDE, I. 1963. «Les états dysharmoniques graves, étude clinique de formes précoces intriquant relation psychotique et symptomatologie de type déficitaire », Psychiatrie de l'enfant, 6.

RICHARD, B. 2005. " Les comportements de scarification chez l'adolescent ", Neuropsychiatrie de l'enfance et de l'adolescence, 53, 3.

SCARAMOZINO, S. 2004. « Pour une approche psychiatrique de l'automutilation : implications nosographiques », Champ psychosomatique, $\mathrm{n}^{\circ} 36$.

SCHARBACH, H. 1986. Automutilations et auto-offenses, Paris, Puf.

SCHARBACH, H. 1987. « Gestes autovulnérants et pratiques auto-offensives », Annales psychiatriques, 2, 4.

Strong, M. 2005. A Bright Red Scream. Self-mutilation and the Language of Pain, London, Virago.

TRICHET, Y. ; LEVY, A. 2008. "Scarification et (auto-)mutilation dans la psychose ", L'information psychiatrique, vol. 84, n 5 .

ZENONI, A. 2009. L'autre pratique clinique, Toulouse, érès.

\section{Résumé}

Les automutilations psychotiques qui surgissent en institution laissent bien souvent les professionnels désemparés, impuissants face à ces passages à l'acte d' « attaque » du corps dans le réel, auxquels ils ne peuvent donner 
sens. Aussi, interroger les coordonnées du déclenchement de tels passages à l'acte s'avère nécessaire et nécessite un examen psychopathologique minutieux de ce qui se présente comme retour du réel du corps dans notre modernité. Au-delà, la clinique de l'automutilation interroge la notion même de corps et du rapport du sujet au monde, à l'autre. L'auteur démontre en quoi le passage de la coupure du corps, à la trace, au trait, voire à l'écriture, sur le corps sont autant de tentatives de «nouage » pour border, faire tenir le corps. Ces écritures du réel sur le corps sont dès lors, dans certains cas, à envisager comme solutions « autothérapeutiques » avec leur effet d'apaisement pour le sujet.

\title{
Mots-clés
}

Passage à l'acte, acting-out, psychose, réel, écriture, processus créatif.

\begin{abstract}
Acts of psychotic self-injury that occur in institutions often leave the staff at loss and powerless when facing such acting outs of the body's "attacks" in the real. The caregivers have trouble giving sense to such acts. It appears fundamental to question the background situation that triggers such an act with a thorough psychopathological study of the body's return of the real, as defined by modernity. Beyond this point, the clinical study of self-harm questions the idea of a body itself and the individual's relationship to the world, to the other. The author shows how the transition from cutting the body to a trace, a line or even writing on the body can be an attempt to "knot together" the real, symbolic and imaginary so as to place the body within its boundaries. These inscriptions of the real on the body are, in certain cases, to be considered as "auto-therapeutic" solutions with their calming effects for the patient.
\end{abstract}

\section{Keyworks}

acting out, psychosis, real, writing, creative process. 